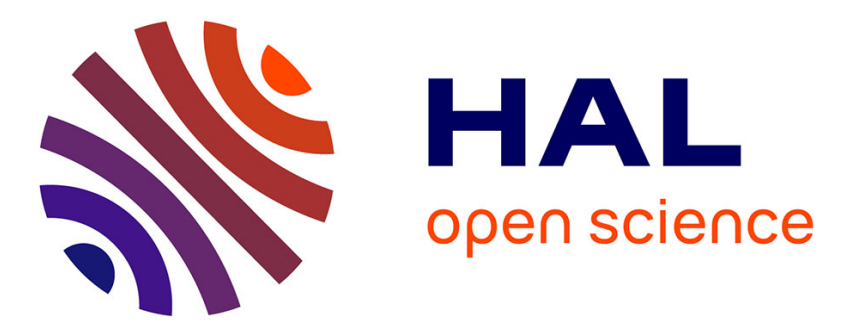

\title{
Lifestyle Agent: The Chat-Oriented Dialogue System for Lifestyle Management
}

\author{
Hayaki Kawata, Kazuki Ookawara, Masahumi Muta, Soh Masuko, Jun’ichi
} Hoshino

\section{- To cite this version:}

Hayaki Kawata, Kazuki Ookawara, Masahumi Muta, Soh Masuko, Jun'ichi Hoshino. Lifestyle Agent: The Chat-Oriented Dialogue System for Lifestyle Management. 16th International Conference on Entertainment Computing (ICEC), Sep 2017, Tsukuba City, Japan. pp.396-399, 10.1007/978-3-31966715-7_48. hal-01771241

\section{HAL Id: hal-01771241 \\ https://hal.inria.fr/hal-01771241}

Submitted on 19 Apr 2018

HAL is a multi-disciplinary open access archive for the deposit and dissemination of scientific research documents, whether they are published or not. The documents may come from teaching and research institutions in France or abroad, or from public or private research centers.
L'archive ouverte pluridisciplinaire HAL, est destinée au dépôt et à la diffusion de documents scientifiques de niveau recherche, publiés ou non, émanant des établissements d'enseignement et de recherche français ou étrangers, des laboratoires publics ou privés.

\section{(c)(1)}

Distributed under a Creative Commons Attribution| 4.0 International License 


\title{
Lifestyle Agent : The Chat-oriented Dialogue System for Lifestyle Management
}

\author{
Hayaki Kawata ${ }^{1 *}$, Kazuki Ookawara ${ }^{1}$, Masahumi Muta ${ }^{2[0000-0001-5795-0603]}$, \\ Soh Masuko ${ }^{2[0000-0002-3277-5690]}$ and Jun'ichi Hoshino ${ }^{1}$ \\ ${ }^{1}$ University of Tsukuba, Graduate school of Systems and Information Engineering, \\ 1-1-1, Tennodai, Tsukuba-shi, Ibaraki, Japan \\ \{kawata.hayaki, okawara.kazuki \}@entcomp.esys.tsukuba.ac.jp, \\ jhoshino@esys.tsukuba.ac.jp \\ ${ }^{2}$ Rakuten, Inc., Rakuten Institute of Technology, \\ Rakuten Crimson House, 1-14-1, Tamagawa, Setagaya-ku, Tokyo, Japan \\ \{masafumi.muta, so.masuko\}@rakuten.com
}

\begin{abstract}
In this paper, we propose Lifestyle Agent, which manages user's lifestyle in a chat format. Lifestyle agent counsels users through chat and encourages the formation of motivation with the aim of improving users' lifestyle habits. In an evaluation experiment, we had users experience chat examples with Lifestyle Agent and gained knowledge on dialogue planning to promote improvement of lifestyle habits.
\end{abstract}

Keywords: Lifestyle improvement, Chat communication, Rule-based dialogue management

\section{Introduction}

Because lifestyle diseases account for the majority of domestic medical expenses in Japan, the Japanese government has promoted the prevention of lifestyle diseases of citizens [1]. Lifestyle-related diseases can be prevented by improving unfavorable daily lifestyle habits. However, because the daily internal changes due to lifestyle habits are extremely small, It is difficult to manage lifestyle habits with awareness of risks that lie a few years ahead. For lifestyle-related diseases caused by social stress, mental health care such as counseling is important.

In this paper, we propose a Lifestyle Agent that can is designed to improve lifestyle while enjoying communication. Lifestyle Agent performs user counseling through chat: the system asks questions about the user's lifestyle and health consciousness, gives advice appropriate to the user's lifestyle, provides support for improving lifestyle habits, etc. Users can improve their lifestyle habits while enjoying communication by counseling that is based on their own lifestyle habits. In evaluation experiments, we had users experience chat examples with the Lifestyle Agent, and gained insight on dialogue planning to make lifestyle habit improvement more effective and enhance the chat sustainability of users. 
Chat systems are recently attracting attention in the dialogue field [2][3]. The chat dialogue is treated as a non-target task: it is important to let the dialogue with the user continue longer. This seems to include the element of enjoying the dialogue itself. In this paper, we consider the dialogue system as a representation of entertainment products.

\section{System Overview}

This system is a chat-style dialogue system that is aimed at improving the lifestyle habits of users along with the agents. Fig.1 shows the operation of the system. The knowledge database of this system is cited from the policies of the Japanese government posted online to prevent lifestyle-related diseases. Of these, "diet," "sleep," "alcohol consumption," "smoking," etc. are mentioned regarding measures for prevention of lifestyle diseases, and information on these four items is used in the dialogue.

Using the buttons at the bottom of the screen, users can talk to the system to encourage communication and record diet and sleep. Based on the user profile and the action history of the user, a common basis for dialogue is constructed, and the system provides knowledge suitable for each user and feedback based on it. The user can live a physically and mentally healthy life by acquiring correct knowledge and lifestyle habits.

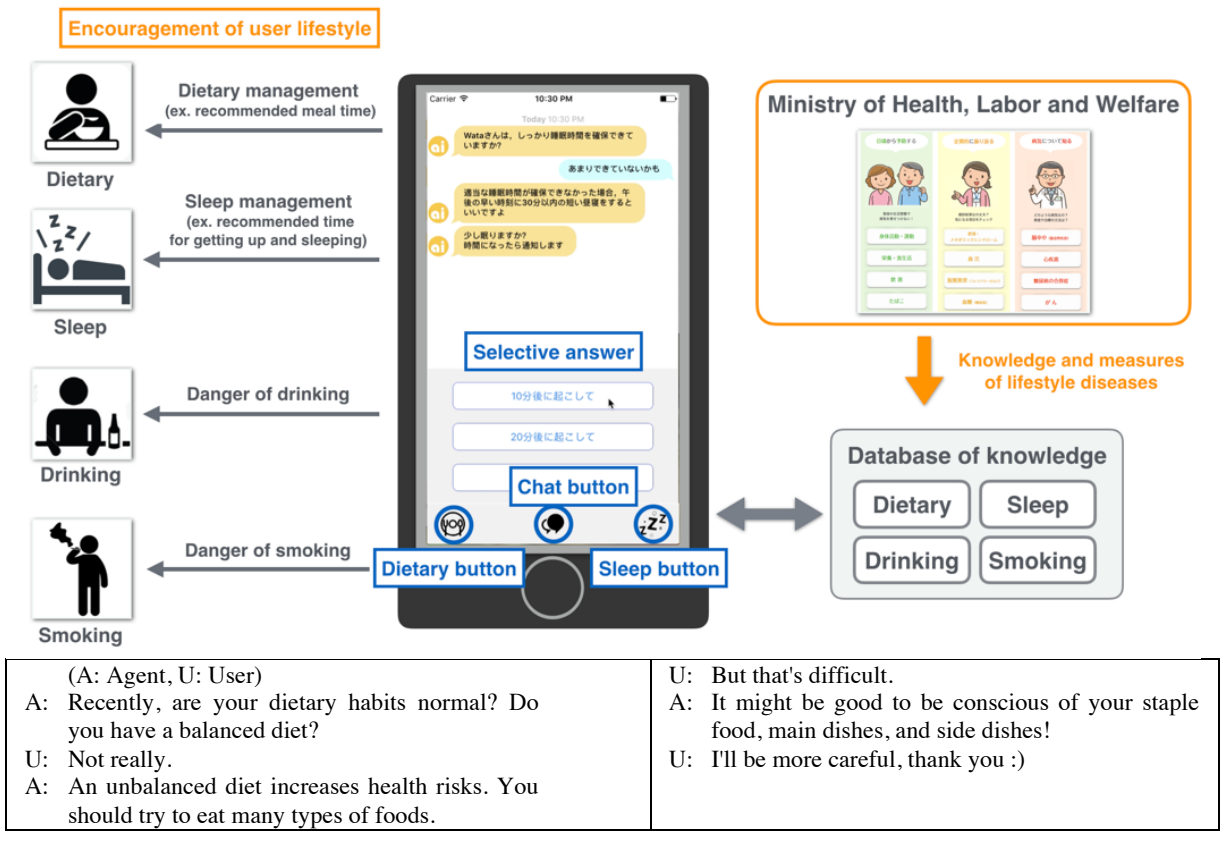

Fig. 1. Example of chat usage and system configuration in the proposed system. 


\section{Experiments}

In constructing such a system, we examined what effect contents related to the user and contents not related to the user have on the user's interest/consciousness. As an impression evaluation experiment, we conducted an investigation of the impressions of persons who experienced the dialogue. A total of 12 subjects $(11$ men and 1 woman; subjects were in their 20s) experienced chat examples through the application, and we investigated changes in impression and consciousness in subjects.

Based on the results of the preliminary questionnaire, we classified subjects in Table 1 by the categories of eating habits, sleeping habits, alcohol consumption, and smoking. Here, the number represents the number of persons. Results of totaling the questionnaire results under this category are shown in Figs. 2 and 3. Fig.2 shows the degree of interest in lifestyle diseases before and after the experiment and the change in sense of crisis; Fig. 3 shows the feeling of crisis felt by persons in each category in response to the dialogue of each category.

In Fig. 2, in the group without dietary consciousness and the group with smoking habits, there was a significant difference in the improvement of the degree of interest after the experiment. Moreover, in Fig.3, differences were noted not only concerning categories related to the groups, but also concerning the feeling of sense of crisis for other categories.

These results revealed that it is effective to divide the user type by diet, alcohol consumption, and smoking habits. By constructing dialogue planning based on these user types, it will be possible to increase the value of the user experience in the proposed system.

Table. 2 Criteria of classification of subjects and number of persons in each

\begin{tabular}{|c|c||c|c||c|c||c|c|}
\hline \multicolumn{2}{|c||}{ Dietary consciousness } & \multicolumn{2}{c||}{ Sleeping consciousness } & \multicolumn{2}{c||}{ Dringking frequency } & \multicolumn{2}{c|}{ Smoking habits } \\
\hline Yes & No & Yes & No & High & Low & Yes & No \\
\hline 5 & 7 & 5 & 7 & 6 & 6 & 6 & 6 \\
\hline
\end{tabular}
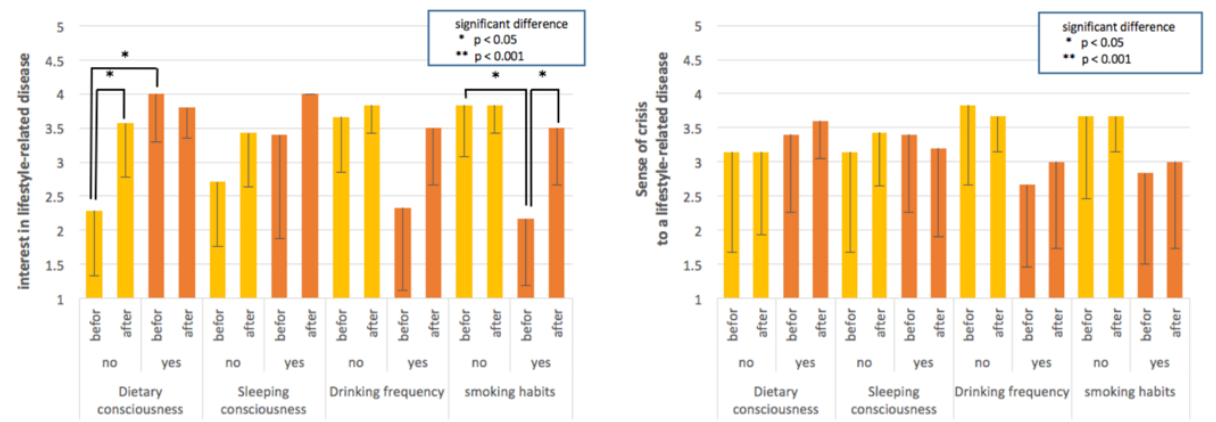

Fig. 2 Changes in interest level (left) and sense of crisis (right) before and after experiment 

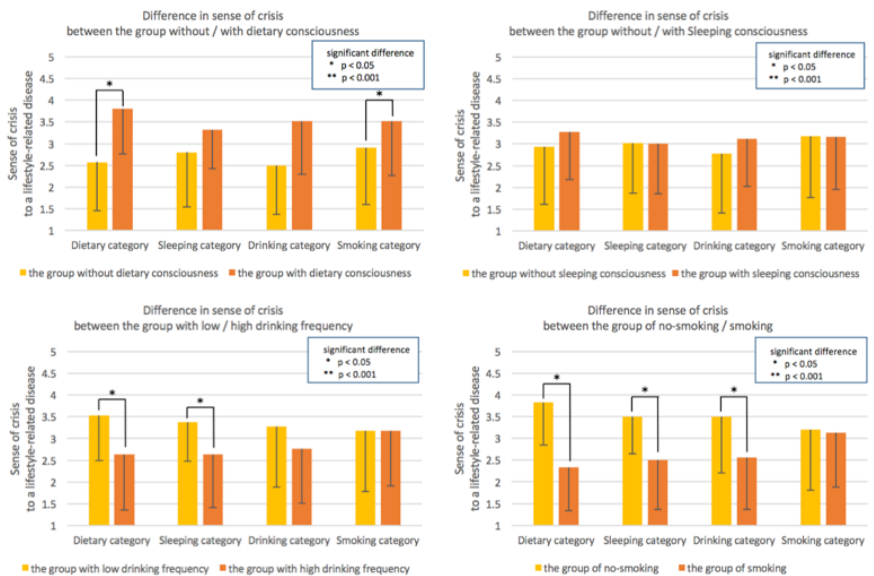

Fig. 3 Sense of crisis felt for each category of dialogue as seen by classification of lifestyle consciousness

\section{Conclusion}

In this paper, we propose a Lifestyle Agent that manages lifestyle habits of users through chat-style communication. The proposed system understands the user's lifestyle and conducts guidance for improvement based on dialogue. The user can review (be conscious of) his or her own lifestyle habits while enjoying communication through dialogue with the Agent, who tries to connect to his or her life.

The results of the evaluation experiment revealed that it is important to differentiate the user type using three criteria (diet, alcohol consumption, smoking) in order to make the user conscious of his or her own lifestyle. In the proposed system, it is possible to increase the value of the user experience by changing the dialogue contents for each of these user types.

Lifestyle Agent is a dialogue system that can walk the same time as the user. In the future, we would like to make products with more entertainment elements by using various information from the users' daily lives.

\section{References}

1. "Welcom to Ministry of Health, Labour and Welfare", http://www.mhlw.go.jp/english/index.html, 2017-03-30.

2. Banchs, Rafael E., and Haizhou Li. "IRIS: a chat-oriented dialogue system based on the vector space model." Proceedings of the ACL 2012 System Demonstrations. Association for Computational Linguistics, 2012.

3. Lasguido, N. I. O., et al. "Utilizing human-to-human conversation examples for a multi domain chat-oriented dialog system." IEICE TRANSACTIONS on Information and Systems 97.6 (2014): 1497-1505. 